\title{
PREFERENCIAS Y DISPOSICIÓN A PAGAR POR UVA DE MESA ORGÁNICA EN LA REGIÓN DEL MAULE, CHILE ${ }^{1}$
}

\author{
ARCADIO ALBERTO CERDA URRUTIA², LEIDY YOMARY GARCÍA PÉREZ ${ }^{3}$, \\ JOHANNA CAROLINA GONZÁLEZ CANCINO ${ }^{4}$, \\ ASHLEY IGNACIO SALVATIERRA NÚÑEZ
}

RESUMEN- El presente estudio tuvo como objetivo determinar las preferencias y disposición a pagar por consumo de uva de mesa orgánica. Específicamente, se buscó determinar la utilidad y jerarquizar los atributos más importantes que consideran los individuos al momento de la elección de uva de mesa y determinar la disposición a pagar por uva de mesa orgánica en la Región del Maule, Chile. Se aplicó una encuesta a una muestra probabilística y estratificada por niveles socioeconómico en los hogares en los principales centros urbanos de la región. Se utilizó el método de análisis conjunto a fin de determinar las preferencias de los individuos por los distintos atributos y el método de valoración contingente para determinar el monto medio de sobreprecio que una persona está dispuesta a pagar por un kilo de uva orgánica. Igualmente se calculó la disposición a pagar marginal vía el método de análisis conjunto. Se determinó que el atributo más importante a la hora de adquirir este bien es el precio, mientras que la mezcla de atributos que más tiene utilidad por orden de importancia es el sabor dulce, de producción orgánica, que no posea semillas y de piel delgada. Además, se estimó que las personas están dispuestas a pagar un sobreprecio por la uva de mesa orgánica, abriendo nuevas oportunidades de negocios para el sector frutícola.

Términos para indexación: producción ecológica, valoración contingente, análisis conjunto.

\section{PREFERENCIAS E DISPOSIÇÃO EM PAGAR POR UVA DE MESA ORGÂNICA NA REGIÃO DE MAULE, CHILE}

RESUMO- O presente estudo teve como objetivo determinar as preferências e disposição em pagar por consumo de uva de mesa orgânica. Especificamente, buscou-se determinar a utilidade e hierarquizar os atributos mais importantes que os indivíduos consideram no momento da escolha de uva de mesa e determinar a disposição em pagar por uva de mesa orgânica na Região de Maule, Chile. Aplicou-se uma pesquisa a uma amostra probabilística e estratificada por níveis sócio-econômicos nos lares, nos principais centros urbanos da região. Utilizou-se o método de análise conjunto a fim de determinar as preferências dos indivíduos pelos diferentes atributos e o método de valorização contingente para determinar o montante médio de sobrepreço que uma pessoa está disposta a pagar por um quilo de uva orgânica. Igualmente calculou-se a disposição marginal em pagar via método de análise conjunto. Determinou-se que o atributo mais importante na hora de adquirir este bem é o preço, enquanto que a mistura de atributos que mais tem utilidade por ordem de importância é o sabor doce, de produção orgânica, que não possua sementes e de casca fina. Além disso, estimou-se que as pessoas estão dispostas a pagar um sobrepreço pela uva de mesa orgânica, abrindo novas oportunidades de negócios para o setor frutícola.

Termos para indexação: produção ecológica, valorização contingente, análise conjunto.

\footnotetext{
1(Trabalho 222-10). Recebido em: 25-10-2011. Aceito para publicação em: 03-05-2011.

${ }^{2}$ Ph.D., MBA, MSc. Ing. Com. Director Centro de Desarrollo Empresarial, Facultad de Ciencias Empresariales, Universidad de Talca, ${ }^{3}$ Norte 685, Talca, Chile. Email: acerda@utalca.cl

${ }^{4}$ Estudiante de Doctorado en Economía, Universidad de Chile, MBA, Economista, Becaria CONICYT. Dirección postal: La Macarena 101, Las Condes, Chile, Email: legarcia@fen.uchile.cl.

${ }_{5}^{5}$ Ingeniero Comercial, Universidad de Talca, 2 Norte 685, Talca, Chile, Email: joa_joi@hotmail.com

${ }^{6}$ Ingeniero Comercial, Universidad de Talca, 2 Norte 685, Talca, Chile, Email: neverdie1@gmail.com
} 


\section{INTRODUCCIÓN}

En Chile es escasa la información respecto a las preferencias de los consumidores y disposición a pagar por productos agrícolas orgánicos, y menos frutícolas, a pesar de los estudios que existen en países más desarrollados (NADEZHDA; MAZZOCCO, 2008; BOCCALETT; NARDELLA, 2000; BAKER, 1999). Específicamente, trabajos que identifiquen las preferencias de los consumidores por distintas características de uva de mesa son aún más escasos (PIVA et al., 2006), aunque en la literatura se encuentran artículos que relacionan a uvas orgánicas en la producción y preferencias por vino (CERDA, 2010; SANCHEZ;GIL, 1998). La falta de estudios relacionados también se refleja en la casi inexistencia de trabajos que busquen determinar la disposición a pagar (DAP) por la uva de mesa orgánica.

Uno de los aspectos más relevantes en investigación de mercado es determinar las preferencias de los consumidores de tal forma que los productores puedan adaptarse a dichas preferencias. Esto tiene mayor relevancia cuando los agronegocios deben actuar en una economía global cada vez más competitiva donde los requerimientos ambientales son cada vez más exigentes sobre la actividad (CERDA et al., 2010).

Uno de los pocos estudios que relaciona los atributos que buscan los consumidores en el consumo de uva es el desarrollado por Piva et al. (2006), quienes basados en una encuesta realizada en Madrid, donde se identifican los atributos de la uva ideal, a partir de tablas de frecuencias, aporta información base relevante para futuros estudios, a pesar de constituir una limitante para el análisis estadístico.

El objetivo de este estudio es determinar las preferencias y disposición a pagar por consumo de uva de mesa orgánica. Específicamente, se busca determinar la utilidad y jerarquizar los atributos más importantes que consideran los individuos al momento de la elección de uva de mesa, vía el método de análisis conjunto (MAC) y determinar la disposición a pagar de los consumidores por uva de mesa orgánica usando el método de valoración contingente (MVC) y MAC.

La presente investigación pretende hacer un aporte adicional en término de información sobre las variedades de uva que se pueden fomentar y producir en base a las preferencias de los consumidores, con el propósito de aumentar el consumo de la uva de mesa.

\section{MATERIALES Y METODOS}

\section{Diseño de la investigación}

La información utilizada en este estudio se obtuvo de la aplicación de dos instrumentos; una pre-encuesta dirigida y la encuesta final. El objetivo de la pre-encuesta fue verificar si los atributos o características deseables de la uva de mesa de parte de los consumidores coincidían con los pre-establecidos, basados en el estudio de Piva et al. (2006), o si existía algún otro atributo que se considerase relevante en la decisión de compra. Además, se realizó un primer sondeo acerca de la DAP por uva orgánica, valores con los cuales posteriormente se construyó el vector de pago (entendiéndose como los valores de sobreprecio hipotético por adquirir un kilo de uva orgánica con respecto a uva convencional) con sus respectivos limites inferiores y superiores, de acuerdo a lo requerido por el MVC en su formato dicotómico doble.

El instrumento de medición tenía cuatro secciones: la primera incluyó introducir al individuo en el proceso producción orgánica y tradicional, y preguntas sobre consumo de uva, lugar de compra, disponibilidad, calidad y precio pagado regularmente. La segunda consideró preguntas sobre DAP por uva orgánica. La tercera, consideró preguntas sobre las preferencias de los individuos con respecto a diversos productos hipotéticos de uva, previamente generados mediante el diseño ortogonal proporcionado por el software estadístico SPSS versión 15.0. En la primera parte de esta sección el encuestado debe "jerarquizar" de mayor a menor importancia (1 a 8) los diferentes productos hipotéticos de acuerdo a sus preferencias, mientras que en la segunda parte debió "calificar" los productos hipotéticos presentados de acuerdo a una escala de evaluación de 1 a 7 (1 mínima y 7 máxima). La cuarta sección recabó información de tipo demográfica y socioeconómica.

El instrumento de medición se aplicó en forma directa por parte de los investigadores y personal de apoyo en los hogares de los principales centros urbanos (Ciudad de Talca, Curicó, Linares, y Cauquenes) de la región del Maule, Chile. La muestra es de carácter probabilística estratificada por nivel socioeconómico de la población.

\section{Métodos para analizar las preferencias y disposición a pagar}

Los métodos utilizados para estimar la DAP fueron el MVC y el MAC.(Sanchez et al., 2001; Umberger et al. 2002). Método de análisis conjunto. El MAC es un método multivariado que desagrega la estructura de las preferencias de los consumido- 
res en valores de utilidad (Padilla et al., 2007). Las utilidades asignadas a cada uno de los atributos del producto se obtienen mediante la descomposición de las valoraciones globales realizadas por los sujetos entrevistados sobre los diferentes productos hipotéticos que se les ofrecen (Green \& Srinivasan, 1978).

En general, la función de utilidad se expresa como $\mathrm{U}=\mathrm{U}\left(\mathrm{Z}_{\mathrm{t}}\right)$, donde $\mathrm{Z}_{\mathrm{t}}$ es el valor de un vector de atributos para la característica $t$ de las posibilidades de elecciones que el consumidor pretende adquirir maximizando su utilidad, para lo cual comúnmente se usa una función de utilidad lineal (Green \& Srinivasan, 1978; Steenkamp, 1987).

Considerando los cinco atributos evaluados en este estudio, el modelo econométrico a estimar se puede expresar como:

$$
R_{i}=\beta_{0}+\sum_{i=1}^{3} \beta_{1 i} D_{1 i}+\sum_{j=1}^{2} \beta_{2 j} D_{2 j}+\sum_{k=1}^{2} \beta_{3 k} D_{3 k}+\sum_{l=1}^{2} \beta_{4 l} D_{4 l}+\sum_{m=1}^{2} \beta_{5 l} D_{5 l}+\varepsilon_{i}
$$

donde Ri, es la valoración, obtenida a partir de la "jerarquización” (Modelo MAC1) de productos (1 a 8) y la "calificación" (Modelo MAC2) de productos (desde 1 a 7 niveles), dependiendo del modelo a estimar. Los coeficientes $\beta_{1 i},, \beta_{2 j} \beta_{3 k} \beta_{4 l} \beta_{5 m}$ están asociados a las característica de cada atributo, $\mathrm{i}=$ precio (tres), $\mathrm{j}=$ sistema de producción (no orgánico y orgánico), $\mathrm{k}=$ sabor (más o menos dulce), and l=piel (delgada y gruesa), $\mathrm{m}=$ semilla (con o sin semilla).

Los niveles del atributo precio, se basan en un promedio de los veinte valores inferiores, medios y superiores de la pre-encuesta. Se seleccionaron cinco atributos con los respectivos niveles, lo que entrega 48 combinaciones para el análisis experimental completo. Para reducir el número de combinaciones a un número factible de analizar por parte del entrevistado, se generó un diseño ortogonal, el que arrojó ocho combinaciones de productos hipotéticos a analizar (Tabla 1). Para poder obtener la utilidad se le pide al encuestado que "jerarquice" y "califique" las ocho tarjetas de acuerdo a sus preferencias.

La DAP marginal (DAPm) para cada atributo puede ser calculada por la razón entre el coeficiente del atributo en cuestión estimado, dividido por el valor negativo del coeficiente de precio estimado (GAN; LUZAR, 1993), es decir, la DAPm por uva orgánica según el MAC, se obtiene por la razón entre la utilidad de uva orgánica dividido por el coeficiente de precio estimado. Igualmente, el MAC permite calcular la influencia de cada atributo en la utilidad del consumidor (GREEN; WIND, 1975). Así el índice de utilidad relativa (IUR) de cada atributo es calculada de acuerdo a Halbrendt et al. (1995), usando la expresión: $\mathrm{IUR}_{\mathrm{i}}=100 *$ Rango Utilidad $1 / \Sigma$ Rangos de todos los atributos].
Valoración Contingente. CV es comúnmente usado para valorar bienes ambientales ya que permite encontrar la DAP debido a un cambio en un bien ambiental usando mercado hipotéticos (CERDA et al., 2007). Sin embargo, también ha sido usado para estimar DAP por cambios en los atributos de los alimentos (MAYNARD; FRANKLIN, 2003; CAMPICHE et al., 2004) y para valorar la DAP por fruta y vegetales libres de pesticidas (BOCCALETTI; NARDELLA, 2000) y productos orgánicos (CERDA, 2010; GIL et al., 2000).

El modelo usado se basa en el modelo de diferencia en la función indirecta de utilidad (HANEMANN, 1984). Dada la función de utilidad $u_{j}=v_{j}\left(P, I ; q_{j}\right)+\varepsilon_{j} ; \quad$ donde $\mathrm{j}=0$ representa situación inicial (sin cambio en las características del bien), $\mathrm{j}=1$ situación final (casos de uva orgánica), $P$ es el precio de la uva de mesa, I es el Ingreso, $\mathrm{q}$ representa los atributos de la uva, entre otros, y $\varepsilon$ es el término de error. Individuos quienes desean consumir uva de mesa orgánica necesitan pagar un sobre precio $b_{t}$, donde $t$ representa diferentes sobreprecios que enfrentan los entrevistados. Por lo tanto, la probabilidad de pagar por uva orgánica viene dado por $\operatorname{Pr}($ yes $)=\operatorname{Pr}\left[v_{1}\left(P, I-b_{t} ; q_{1}\right)+\varepsilon_{1}>v_{0}\left(P, I ; q_{0}\right)+\varepsilon_{0}\right]$ o alternativamente, $\operatorname{Pr}($ yes $)=\operatorname{Pr}(\Delta v>\eta)=F_{\eta}(\Delta v)$ con $\eta \equiv \varepsilon_{0}-\varepsilon_{1} . F_{\eta}$ representa la función de distribución acumulada de $\eta$, donde para este estudio se asume logística.

La diferencia entre dos funciones indirectas de utilidad lineal, para una situación con y sin uva orgánica se puede expresar como $\Delta v=\alpha-\beta b_{t}$. En este caso, para mejorar la eficiencia de los estimadores, se utiliza el MVC en su formato dicotómico doble.

Para estimar los coeficientes se usa el Programa CVM (COOPER; HELLERSTEIN, 1994). Este programa se basa en la estimación de la función de máxima verosimilitud relevante analizando la primera y segunda derivada de acuerdo a Hanemann et al. (1991). La DAP adicional por un $\mathrm{kg}$ de uva uva orgánica se estima por la media, definida como, $E(C)=C^{*}=\alpha / \beta$ que representa la esperanza de la verdadera DAP adicional C (BOYLE; BISHOP, 1998; VÁSQUEZ et al., 2007).

Los intervalos de confianza alrededor de la media de la DAP fueron calculados mediante el enfoque de Krinsky \& Robb (PARK et al., 1991).

Para determinar el vector de pago (valores de sobreprecio por $\mathrm{kg}$ ), se usaron los precios máximos de pago obtenidos de la pre-encuesta, a los que se le aplica el método DWEABS, técnica iterativa para encontrar el mínimo error cuadrático medio del diseño de la muestra desarrollado por Cooper (1993). 
El vector de pago se estima con el Programa CVM (COOPER; HELLERSTEIN, 1994), obteniéndose diez valores, con sus respectivos valores inferiores y superiores, para el formato de pregunta dicotómica doble.

\section{RESULTADOS Y DISCUSION}

\section{Características de la población}

La población en estudio son individuos mayores de 18 años de la Región del Maule entrevistados en el mes de mayo de 2010. Para determinar el tamaño de la muestra se asume varianza máxima, un nivel de confianza del 95\% y un error estándar de $4,9 \%$, obteniéndose un tamaño muestral de 398 , dada una población mayor de 18 años en la región del Maule que según el Censo 2002 es de 408.819 habitantes y de acuerdo a la distribución de la población por provincia, se determinó la muestra por ciudad.

\section{Análisis descriptivo de la muestra}

Los entrevistados están compuesto por mujeres $54,3 \%$ y $46,7 \%$ hombres. La edad se distribuye por rangos de entre (18 y 34), (35 y 54) y (sobre 55) en $43,5 \%, 45,2 \%$ y $11,3 \%$; respectivamente. La distribución por ingreso bajo, medio y alto es de $54,7 \%, 37,2 \%$ y $8,1 \%$; respectivamente. Los niveles de educación son: básica $(8,8 \%)$, media $(19,1 \%)$, técnico profesional $(27,1 \%)$, universitaria incompleta $(17,3 \%)$ y universitaria completa $(27,7 \%)$. Los lugares de compra de los entrevistados son ferias libres $(53,5 \%)$, supermercados $(23,4 \%)$, almacenes $(22,2 \%)$ y otros $(1,5 \%)$.

La predisposición a pagar por uva orgánica está directamente relacionada al nivel de ingreso, con una participación, según estrato socioeconómico bajo, medio y alto de $54,6 \%, 68,9 \%$, y $84,3 \%$ respectivamente.

Referente a la elección de productos saludables al momento de realizar una compra, los encuestados declaran que lo hacen solo de vez en cuando (56,3\%), siempre (41\%), y nunca (2,8\%). Al preguntarse por los productos orgánicos el 73,9\% declara conocer los productos de tipo orgánico y el $60,1 \%$ dice haberlos consumido alguna vez. El $51,5 \%$ del total de la muestra asegura que los productos orgánicos son saludables, el 39,4\% piensa que son productos que ayudan al medio ambiente, el $8,8 \%$ cree que son productos que mejoran el estilo de vida.

Bajo el supuesto de que la uva convencional y orgánica tuviesen el mismo precio, el 64,3\% preferiría comprar uva orgánica, mientras que solo el 5,5\% compraría solo uva convencional. El 30,2\% compraría ambos tipos de uva. Además, el 62,3\% de los encuestados se mostraron dispuestos a pagar un monto adicional por kilo de uva de tipo orgánica.

\section{Resultados modelos método valoración contingente}

Para estimar los coeficientes de la DAP se formularon dos modelos, el primero considera solo aquellas personas dispuestas a pagar un sobreprecio por adquirir uva orgánica (Modelo MVC1), mientras que en el segundo modelo se agregaron aquellas personas que por motivos económicos no estaban dispuestas a pagar por uva orgánica, tal como la literatura sugiere, aumentando la muestra en 56 individuos (Modelo MVC2).

Los valores del coeficiente $\beta$ son negativos de ambos modelos, los cuales concuerdan con la teoría, mostrando que los consumidores disminuyen su probabilidad de pago a medida que aumenta el valor del sobreprecio enfrentado (Tabla 2). Para el Modelo MVC1 la DAP es \$500,3 (US\$1.06, al tipo de cambio de abril 8 de 2011) adicionales por kilo. $\mathrm{Si}$ se incorporan aquellas personas que se negaron pagar por razones económicas (MVA2) dicho valor, es $\$ 405,6(\mathrm{US} \$ 0,86)$ adicional por kilo (Tabla 2).

\section{Resultados modelo de método análisis conjunto}

La importancia relativa de los atributos para el Modelo MAC1 (modelo de jerarquización) es precio $(28,78 \%)$, sabor $(23,61 \%)$, tipo de producción $(18,15 \%)$, semilla $(15,30 \%)$ y tipo de piel $(13,1 \%)$. Donde los niveles preferidos para dichos atributos de la uva son dulce, producción orgánica, sin semilla y piel delgada (Tabla 3). Las utilidades entregadas por Modelo MAC2 (modelo de calificación), no observan cambios significativos con el análisis anterior, ya que las utilidades se distribuyen de igual forma en cada nivel $(28,77 \%, 22,23 \%, 18,01 \%, 15,99 \%, 15,00 \%)$; sin embargo se observan algunos cambios en los valores de utilidad.

En el Modelo MAC1, el producto hipotético que mayor utilidad entregó fue el quinto con una utilidad de 5,85; en tanto que los niveles de los atributos preferidos que posee son piel delgada, sin semillas, de sabor dulce cuya producción es de tipo orgánica y que el kilo de uva de este tipo sea aproximadamente $\$ 883$ (US\$1.87). Por otro lado, el Modelo MAC2 arrojó que el producto hipotético preferido sigue siendo el quinto producto con la misma mezcla de atributos, pero con una utilidad levemente superior de 6,11.

En cuanto al orden de preferencia de los ocho 
productos hipotéticos en ambos modelos se observa el mismo orden de los productos, lo que permite inferir cierto grado de congruencia en el orden que se les entregaba a los productos y a la evaluación que se les hacía a los mismos por parte de los encuestados. El coeficiente precio tiene signo negativo, lo cual es congruente con la teoría económica, que menciona que a mayor precio menor es la disposición a pagar.

Los estadísticos R de Pearson y Tau de Kendall muestran que existe una alta correlación en las preferencias.

En ambos modelos se observa que el atributo más importante es el precio a la hora de decidir si comprar o no un producto, lo que corrobora los resultados obtenidos por Sánchez et al. (2001). De los cinco atributos el de menor importancia es la piel para ambos modelos, distinto a lo encontrado por Piva et al. (2006), en donde la piel está en segundo lugar de importancia.

Luego del análisis de ambos métodos se llegó a determinar que los individuos tienen una DAP media positiva por uva orgánica, mientras DAP marginal obtenida en base a MAC tiende a ser cercano a cero (Tabla 2 y Tabla 3). Esta diferencia no necesariamente refleja que un análisis sea mejor que otro, dado que son dos enfoques distintos que se aplican con diferente metodología. Además, una provee una estimación media y la otra una marginal, dando un rango de variación a considerar por los productores.

TABLA 1 - Diseño Ortogonal para análisis de preferencias por uva de mesa

\begin{tabular}{cllllc}
\hline Tarjeta & Sabor & Semillas & Tipo de Piel & Producción & Precio \\
\hline 1 & Agridulce & Con Semillas & Gruesa & Convencional & $\$ 883$ \\
2 & Agridulce & Sin Semillas & Gruesa & Orgánica & $\$ 525$ \\
3 & Dulce & Con Semillas & Delgada & Convencional & $\$ 525$ \\
4 & Agridulce & Sin Semillas & Delgada & Convencional & $\$ 1413$ \\
5 & Dulce & Sin Semillas & Delgada & Orgánica & $\$ 883$ \\
6 & Dulce & Con Semillas & Gruesa & Orgánica & $\$ 1413$ \\
7 & Agridulce & Con Semillas & Delgada & Orgánica & $\$ 525$ \\
8 & Dulce & Sin Semillas & Gruesa & Convencional & $\$ 525$ \\
\hline
\end{tabular}

Fuente: Elaboración propia.

TABLA 2 - Resultados modelos método de valoración contingente.

\begin{tabular}{lcc}
\hline \multicolumn{1}{c}{ Variables } & Modelo MVC1 & Modelo MVC2 \\
\hline Constante $=\alpha$ & 2,8588 & 1,8631 \\
$\beta$ & $(0,2428)^{*}$ & $(0,1802)^{*}$ \\
& $-0,0057$ & $-0,0045$ \\
$\eta$ & $(0,0005)^{*}$ & $(0,0004)^{*}$ \\
Log likelihood & 248 & 304 \\
DAP media** & $-327,15$ & $-408,90$ \\
Intervalo de confianza 95\% & $\$ 500,3$ & $\$ 405,4$ \\
\hline
\end{tabular}

Valores entre paréntesis indican el error estándar.

**Pesos chilenos adicionales por kilo de uva de mesa

Tipo de cambio observado $\$ / 1 \mathrm{USD}=549,17$ al 20 mayo 2010, Banco Central de Chile.

Fuente: Elaboración propia 
TABLA 3 - Utilidades estimadas modelos método análisis conjunto

\begin{tabular}{cccc}
\hline Atributo & Nivel & Modelo MAC1 & Modelo MAC2 \\
\hline Sabor & Dulce & 0,6564 & 0,3175 \\
& Agridulce & $-0,6564$ & $-0,3175$ \\
Semilla & Con semillas & $-0,3166$ & $-0,1862$ \\
& Sin semillas & 0,3166 & 0,1862 \\
Producción & Convencional & $-0,3649$ & $-0,2164$ \\
& Orgánicas & 0,3649 & 0,2164 \\
Piel & Delgada & 0,2249 & 0,1856 \\
& Gruesa & $-0,2249$ & $-0,1856$ \\
Precio & $\$ 525$ & $-0,8506$ & $-0,3948$ \\
& $\$ 883$ & $-1,7012$ & $-0,7896$ \\
& $\$ 1.413$ & $-2,5519$ & $-1,1844$ \\
Constante & & 5,9886 & 5,8238 \\
& & & \\
\multicolumn{2}{c}{ Coeficiente Precio } & $-0,8510$ & $-0,3950$ \\
DAPmg*** & & $\$ 0,43$ & $\$ 0,55$ \\
& R de Pearson & $0,9285^{* *}$ & $0,9949 *$ \\
& Tau de Kendall & & $0,9286 * *$ \\
\hline
\end{tabular}

$* \mathrm{p}=0,000 ; * \mathrm{p}=0,001, \mathrm{MAC} 1$ por jerarquización, MCA2 calificación.

*** Pesos chilenos.

Fuente: Elaboración propia

\section{CONCLUSIONES}

1- Los atributos del producto de uva ideal por orden de importancia son uva dulce, de producción orgánica, sin semilla y con piel delgada.

2- El precio del producto es el atributo más importante a la hora de seleccionar un tipo de uva.

3- Los resultados sugieren que los individuos están dispuestos a pagar un sobreprecio por adquirir uva de mesa de producción orgánica.

\section{REFERENCIAS}

BAKER, G. Consumer preferences for food safety attributes in fresh apples: market segments, consumer characteristics, and marketing opportunities. Journal of Agricultural and Resource Economics, Montana, v.24, p.80-97, 1999.
BOCCALETTI, S.; NARDELLA, M. Consumer willingness to pay for pesticide-free fresh fruit and vegetables in Italy. International Food and Agribusiness Management Review, College Station, v.3, p.297-310, 2000.

BOYLE, K.; BISHOP. R. Welfare measurements using contingent valuation: a comparison of techniques. American Journal of Agricultural Economics, Ames, v.70, n.1, p.20-28, 1988.

CAMPICHE, J.; HOLCOMB, R.; WARD, C. Impacts of consumer characteristics and perceptions on willingness to pay for natural beef in the southern plains. Food Technology Research Report P-1006. Oklahoma Food and Agricultural Products Research and Technology Center, Oklahoma State University, 2004.

CERDA, A.: TORRES, M.; GARCÍA, L. Preferencias y disposición a pagar por vinos orgánicos de parte de los consumidores de la región del Maule, Chile. Revista Panorama Socioeconómico, Talca, v.40, n.28, p.60-71, 2010. 
CERDA, A.; ROJAS, J.; GARCÍA, L. Disposición a pagar por un mejoramiento en la calidad ambiental en el Gran Santiago. Lecturas de Economía, Medellín, v.67, p.143-160, 2007.

COOPER, J. Optimal bid selection for dichotomous choice contingent valuation surveys. Journal of Environmental Economics and Management, New York, v.24, p.25-40, 1993.

COOPER, J.; HELLERSTEIN, D. CVM software. Washington: Resources and Technology Division, 1994.

GAN, CH,; LUZAR, E. A conjoint analysis in waterfowl hunting in Lousiana. Journal of Agricultural and Applied Economics, Baton Rouge, v.25, n.2, p.36-45, 1993.

GIL, J.;. GRACIA, A.; SÁNCHEZ, M. Market segmentation and willingness to pay for organic products in Spain. International Food and Agribusiness Management Review, College Station, v.2, n.3-4, p.207-26, 2000.

GREEN, P.; WIND, J. New way to measure consumers' judgments, Harvard Business Review, Boston, v.53, p.107-117, 1975.

GREEN, P.; SRINIVASAN, V. Conjoint analysis in consumer research: issues and outlook. Journal of Consumer Research, Boston, v.5, p.103-123, 1978.

HALBRENDT, C.; WANG, Q. ; FRAIZ, C., O'DIERNO, L. Marketing problems and opportunities in Mid Atlantic sea food retailing. American Journal of Agricultural Economics, Ames, v.77, p.1313-1318, 1995.

HANEMANN, M.; LOOMIS, J.; KANNINEN, B. Statistical Efficiency of Double-Bounded Dichotomous Choice Contingent Valuation. American Journal of Agricultural Economics, Ames, v.73, p.1255-1263, 1991.

HANEMANN, W. Welfare evaluation in contingent valuation experiments with discrete response. American Journal of Agricultural Economics, Ames, v.66, p.332-341, 1984.

MAYNARD, L.; FRANKLIN, S. Functional foods as a value-added strategy: the commercial potential of'cancer-fighting' dairy products. Review of Agricultural Economics, Thessaloniki, v.25, n.2, p.316-331, 2003.
NADEZHDA K.; MAZZOCCO, M. Consumer preferences and trade-offs for locally grown and genetically modified apples: a conjoint analysis approach. International Food and Agribusiness Management Review, College Station, v.11, n.4, p.31-53, 2008 .

PADILLA, C.; VILLALOBOS, P.; SPILLER, A.; HENRY, G. Consumer preference and willingness to pay for an officially certified quality label: Implications for traditional food producers. Agricultura Técnica, Santiago, v.67, n.3, p.300-308, 2007.

PARK, T; LOOMIS, J.; CREEL, M. Confidence intervals for evaluating benefits estimates from dichotomous choice contingent valuation studies. Land Economics, Wisconsin, v.67, n.1, p.64-73, 1991.

PIVA, C.; LÓPEZ, J; MORGAN, W. The ideal table grapes for the Spanish market. Revista Brasileira de Fruticultura, Jaboticabal, v.28, n.2, p.258-261, 2006.

SÁNCHEZ, M.; GIL, J. Comparación de tres métodos de estimación del análisis conjunto: diferencias en las preferencias en el consumo de vino y en la segmentación del mercado. Estudios de Economía Aplicada, Valladolid, v.10, p.131-146, 1998.

SÁNCHEZ, M.; GRANDE, I.; GIL, J.; GRACIA, A. Diferencias entre los segmentos del mercado en la disposición a pagar por un alimento ecológico: valoración contingente y análisis conjunto. Estudios Agrosociales y Pesqueros, Madrid, v.190, p.141163, 2001.

STEENKAMP, J. Conjoint measurement in ham quality evaluation. Journal of Agricultural Economics, Newcastle, v.38, n.3, p.473-480, 1987.

UMBERGER, W.; FEUZ, D.; CALKINS, C.; KILLINGER-MANN, K. U.S. consumer preference and willingness-to-pay for domestic corn-fed beef versus international grass-fed beef measured through an experimental auction. Agribusiness, San Francisco, v.18, n.4, p.491-504, 2002.

VÁSQUEZ, F.; CERDA, A.; ORREGO, S. Valoración económica del ambiente, Buenos Aires: Thomson Learning, 2007. p.368. 